\title{
A Simple Method to Monitor the Flux of the Neutral Free-Radical Beam Produced by Laser Photo-Deionization of a Negative Ion Beam
}

\author{
Keiji Hayashi, Daisuke Tanaka, Harumi Araki, Tomoki Maruyama, Daisuke Matsumura, \\ Kazuho Toyoda and Noriyuki Tsuji \\ Graduate School of Engineering, Kanazawa Institute of Technology, 7-1 Ohgigaoka, Nonoichi, Ishikawa 921-8501, Japan \\ Fax: 81-76-294-6711, e-mail: khayashi@neptune.kanazawa-it.ac.jp
}

\begin{abstract}
Ion-current difference measurement by light intensity modulation ( ICD ) is introduced as a convenient method to characterize a purified beam of momentum-controlled neutral free radicals produced by photo-deionization of a negative ion beam for the purpose of surface-reaction-selective device processing. The ICD setup developed in this study to estimate the number flux of the photo-deionized neutral particles exhibited the high precision, sensitivity, and spatial resolution.
\end{abstract}

Key words: Neutral Free-Radical Beam, CW Laser, Negative Ion, Quantum Device Processing

\section{INTRODUCTION}

In order to further evolve quantum-functional nano-devices using metastable condensed phases, it has become indispensable in recent years to ingeniously utilize selective surface reactions of labile chemical species such as neutral free radicals for device processing. The problem encountered in the precise experimental study of a chemical reaction between a neutral free-radical species and a well-characterized material surface is how to supply a sufficient-flux purified beam of the momentum-controlled neutral free radicals onto the surface. The continuous supply is all the more difficult, although steady-flux continuous beams are generally more useful for thin-film growth applications than are pulsed ones. Indeed many approaches to produce neutral free-radical beams have been developed also in the fields of experimental chemistry [1], heating of plasmas for controlled thermonuclear fusion, and experimental simulation of low-earth orbit phenomena [2], but efficient production of the refined beam of neutral free radicals ( RBNR ) in steady flux has not been realized yet. In order to overcome the difficulty we have proposed several experimental production approaches, two of which are photo-deionization of negative ion beams ( PDINIB ) $[3,4]$ and photo-dissociation of energetic compound beams ( PDECB ) [5,6].

We have been developing a trial surface processing apparatus utilizing a steady-flux RBNR produced according to the following PDINIB procedure [3]. Firstly, a steady-flux negative ion beam ( NIB ) of the desired chemical species generated by the high current-density negative ion source is purified at the mass separator. Then, immediately after it is decelerated to the desired kinetic energy and made convergent by the triple-electrode decelerator at the inlet of the process chamber, the negative ion beam is passed through the multiple-pass photo-deionizer ( MPDI ), where the negative ions are efficiently neutralized at the NIB waist by photoelectron emission using a folded beam of visible or near-ultraviolet ( UV ) continuous-wave ( $\mathrm{CW}$ ) laser light. Finally, the electrons and the remaining ions in the photo-deionized beam are filtered out electrostatically at the charged-particle filter. The obtained RBNR is applied to the well-defined surface of the sample prepared in the load-lock chamber beforehand. When we apply a neutral free-radical beam produced by the PDINIB method to thin-film growth, a continuous beam obtained using a CW laser is usually more useful than a pulsed beam. However, the power of available CW lasers is generally much weaker than the peak power of pulsed lasers. Thus, how to achieve enhancement of the photo-deionization efficiency is the key point to realize a practical steady-flux beam of neutral free radicals. In the trial PDINIB apparatus, a near-confocal optical resonator composed of a pair of tunable laser optics facing each other is adopted as the MPDI to significantly enhance the photo-deionization efficiency and to minimize the momentum spread of the resultant neutral free-radical particles.

In an application of the PDINIB method to the doping of photo-deionized $\mathrm{Cr}$ atoms during the low-temperature epitaxial growth of (Al,Ga)N by a PDECB-based approach [5], we found a possibility that incorporation of the $\mathrm{Cr}$ atoms into the growing surface of the diluted magnetic semiconductor $(\mathrm{Al}, \mathrm{Ga}, \mathrm{Cr}) \mathrm{N}$ is enhanced by the oblique irradiation. A well-collimated neutral free-radical beam of broad diameter is useful in such a case, to reveal the angular-selective surface-reaction mechanism responsible for the epitaxy.

The purpose of the present study is to develop a convenient technique to monitor the beam flux of the photo-deionized neutral particles incident onto the sample surface, namely, the method of ion-current 
difference measurement by light intensity modulation ( ICD ) applicable as a feedback tool not only to the optimization of the MPDI parameters but also to the control of the RBNR divergence. In this article, after introducing the ICD method, we discuss its performance and applicability based on experiments in comparison with those of the method of photoelectron-current measurement by low-frequency electro-modulation probe ( PMMP ) [3].

\section{THE ICD METHOD}

As long as the beam kinetic energy is so high that the space-charge effects are negligible, we can estimate the beam flux of the photo-deionized neutral particles simply by measuring the change in the NIB current density due to the photodetachment, without applying voltage to the charged-particle filter. In this study, a Faraday cup mounted on a side of the mobile sample holder was used for the monitoring of the NIB current density. The suppressor electrode and the shield cover plate attached to the commercial Faraday cup were

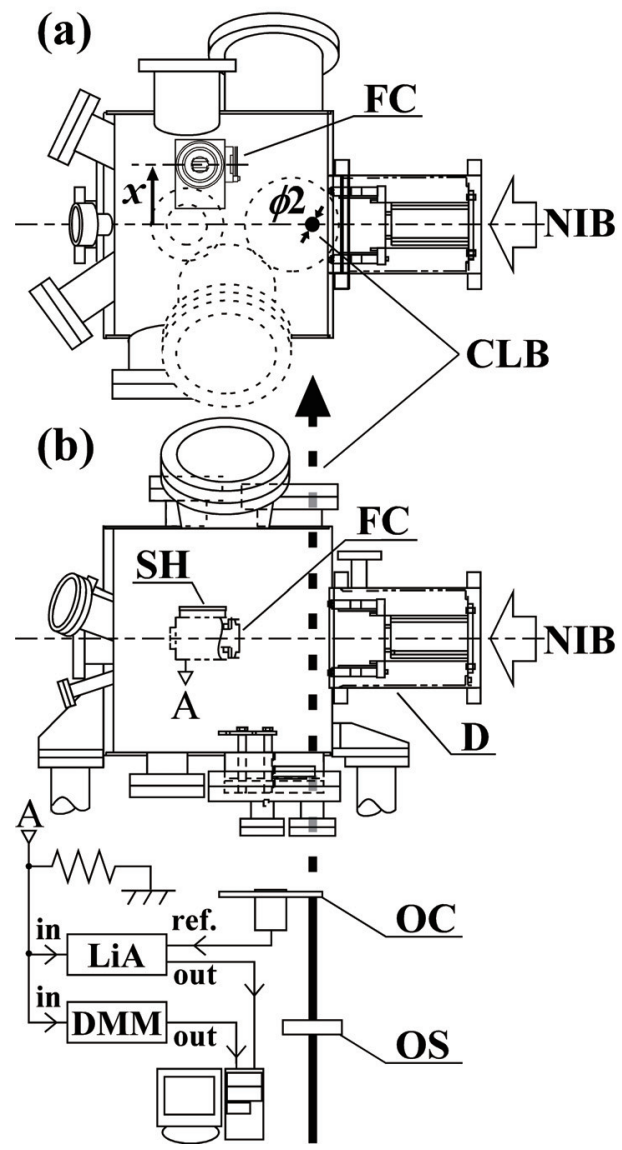

Fig.1. Setup to examine the performance of the ICD method. (a) Top view. (b) Side view with the illustration of the phase-sensitive detection system. NIB: negative ion beam; D: decelerator; SH: sample holder; FC: Faraday cup; CLB: chopped laser light; OS: optical shutter; OC: optical chopper; LiA: lock-in amplifier; DMM: digital multimeter. redesigned to improve the spatial resolution of the RBNR-flux measurement. More specifically, we used a shield cover plate with an aperture whose inner diameter $d_{a}$ is $1.5 \mathrm{~mm}$. Change in the NIB current density synchronous to a mechanically chopped beam of the $\mathrm{CW}$ laser light was measured using a lock-in amplifier. The intensity modulation of the high power $\mathrm{CW}$ light at high frequency up to $2 \mathrm{kHz}$ was performed using a handmade optical chopper. Care was taken not to irradiate the Faraday cup and the suppressor electrode with the laser light.

\section{RESULTS AND DISCUSSION}

Negative ion beams of ${ }^{63} \mathrm{Cu}^{-}$generated by a DC-sputter-type negative ion source were used in the present study. The ${ }^{63} \mathrm{Cu}^{-}$beam of the maximum current more than $1.2 \mathrm{~mA}$ at the beam kinetic energy of $15 \mathrm{keV}$ was extracted reproducibly from the ion source under the optimized condition. We confirmed that the extracted negative particles were not electrons but the ions by measuring the current in between the negative ion source and the mass separator after eliminating electrons from the extracted beam using the source magnet of an interchangeable Freeman type positive ion source.

The precision, the sensitivity, and the spatial resolution of the ICD system were examined using the experimental setup shown in Fig. 1. In order to compare the performances of the ICD and the PMMP methods, we carried out the PDINIB procedure under the condition similar to that of the previous experiment [3] without using the MPDI. A $2 \mathrm{~mm}$-diam, $514.5 \mathrm{~nm}$ visible light beam from a CW Ar ${ }^{+}$ion laser ( Sabre $25 \mathrm{~W}$ ( visible ) $77 \mathrm{~W}$ ( UV ), Coherent, Inc. ) was chopped at $0.5-2.0 \mathrm{kHz}$ and was passed through the process chamber perpendicularly to the horizontal beam of ${ }^{63} \mathrm{Cu}^{-}$ so as to intersect the negative ion beam only once. The distances of the laser beam and the Faraday cup from the outlet of the decelerator ( D in Fig. 1 ) were $20 \mathrm{~mm}$ and $150 \mathrm{~mm}$, respectively, along the NIB axis. At the intersection the diameter of the negative ion beam was about $20 \mathrm{~mm}$. The laser beam was aligned to intersect the NIB axis. More exactly, the alignment was carried out to maximize the photo-deionized-particle flux monitored by the ICD method with the Faraday cup set at the NIB center.

A typical example of the monitored output of the lock-in amplifier is shown in Fig. 2(a). The experiment was carried out under the condition that the NIB kinetic energy was $15 \mathrm{keV}$ and the total NIB current $I_{\text {total }}$ measured in the process chamber without the laser-light irradiation was 11.35 A. Figure 2(b) shows a result of the monitoring continued without the NIB irradiation of the Faraday cup. In both experiments, the laser beam of the light power of $6.06 \mathrm{~W}$ in the process chamber was 


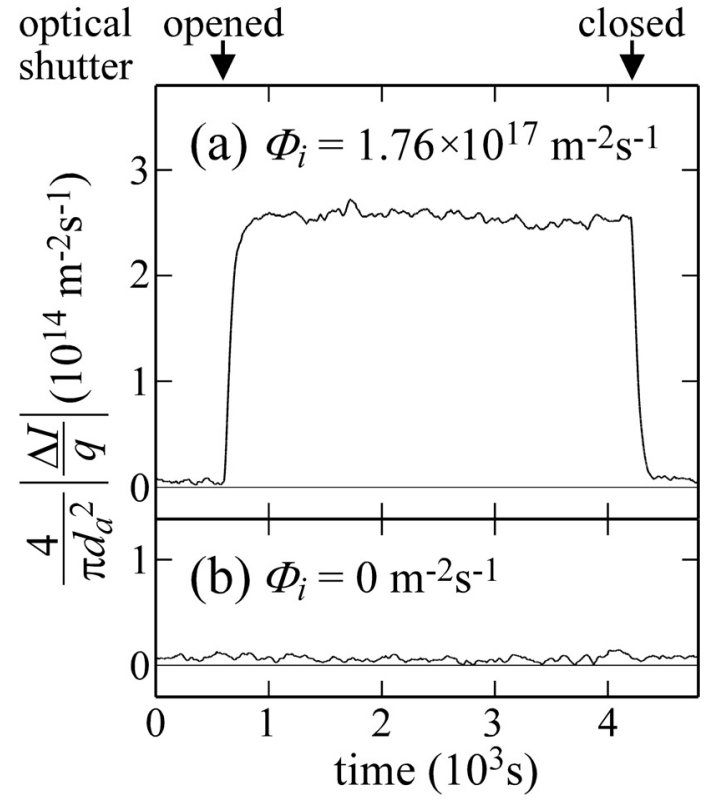

Fig.2. Typical output of the lock-in amplifier monitored (a) with and (b) without the NIB irradiation of the Faraday cup. The chopped laser beam was introduced into the process chamber while the optical shutter was open.

chopped at $1.0 \mathrm{kHz}$. While the optical shutter was open, the negative ion beam was irradiated with the chopped laser beam. The time constant of the lock-in amplifier was $30 \mathrm{~s}$.

The NIB flux ${ }_{i}$ at the Faraday-cup aperture under the dark condition is given by

$$
\Phi_{i}=\frac{4}{\pi d_{a}^{2} \cos \theta}\left|\frac{I}{q}\right|,
$$

where $I$ is the Faraday-cup current measured without the laser-light irradiation and $q$ is the charge of the negative ion. The mean incident angle of the negative ions on the Faraday-cup aperture is approximately $0^{\circ}$ in the present study. In all the experiments, the observed change $I$ in the Faraday-cup current brought about by the laser-light irradiation was a linear function of ${ }_{i}$. The offset $I_{0}$ ( that is to say, the change $I$ measured under the condition that the negative ion beam is interrupted by another mobile Faraday detector at the outlet of the mass separator ) was nonzero only in previous photodetachment experiments using ultraviolet light, thus, is attributed to photoelectron emission from the suppressor electrode or the Faraday cup caused by the scattered laser light. As for the present experiments using the visible light, no appreciable change $I_{0}$ was observed ( for instance, see Fig. 2(b) ). Decrease $\left|{ }_{i}\right|$ in the NIB flux due to the photodetachment is known from

$$
\Delta \Phi_{i}=\frac{4}{\pi d_{a}^{2} \cos \theta}\left|\frac{\Delta{ }_{d}}{q}\right|,
$$

where

$$
\Delta I_{d}=\Delta I-\Delta I_{0} .
$$

We estimated the photo-deionized-particle flux ${ }_{n}$ at the Faraday-cup aperture by

$$
\Phi_{n}=\left|\Delta \Phi_{i}\right|=\frac{4}{\pi d_{a}^{2} \cos \theta}\left|\frac{\Delta l_{d}}{q}\right|,
$$

based on the following consideration. The velocity of a photo-deionized particle is in principle almost equal to that of the negative ion immediately before the photoelectron emission [4]. Moreover, NIB divergence in the process chamber due to space charge effect is negligible as long as the beam kinetic energy is much higher than $100 \mathrm{eV}$. Thus, even if the high-velocity negative ion did not undergo the photoelectron emission, its trajectory in the process chamber would be the same straight one as the photo-deionized particle.

From the experimental result shown in Fig. 2, the time-averaged photo-deionized-particle flux ${ }_{n}$ is estimated based on Eq. (4) to be $\left(257 \pm 0.14 \times 10^{4}\right.$ $\mathrm{m}^{-2} \mathrm{~s}^{-1}$, yielding the photodetachment efficiency defined by

$$
\gamma=\frac{\Phi_{n}}{\Phi_{i}}=-\frac{\Delta l_{d}}{l}
$$

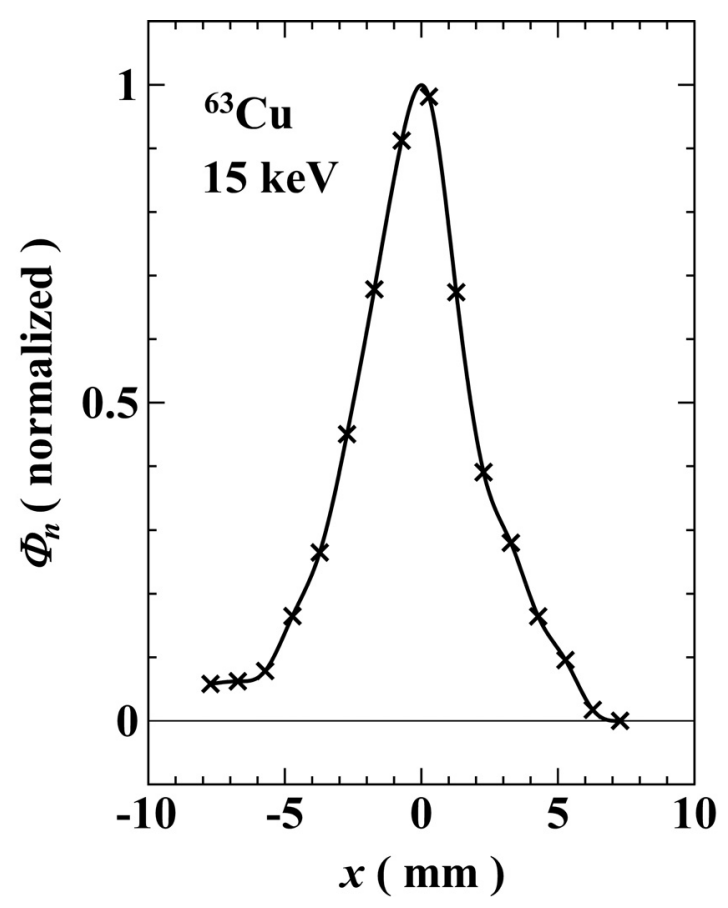

Fig.3. An example of the spatial profile of a neutral free-radical beam at the sample holder of the PDINIB apparatus. The number flux of photo-deionized atomic ${ }^{63} \mathrm{Cu}$ estimated by the ICD method is plotted as a function of the displacement $x$ of the Faraday cup along the horizontal axis perpendicular to the ion beam line. The beam kinetic energy was $15 \mathrm{keV}$. 
of $0.146 \pm 0.00 \varepsilon \%$ without using the MPDI. The signal-to-noise ( $\mathrm{S} / \mathrm{N}$ ) ratio achieved by the ICD method is further improved as the photo-detachment efficiency is enhanced, since the noise mainly results from the fluctuation of the ion-source plasma accidentally synchronized with the light modulation. Under the common conditions of the ion-source plasma and the ion extraction, the magnitude of the noise was proportional to the flux ${ }_{i}$ of the negative ions transported to the Faraday cup, but was independent of the NIB energy and the laser-light power. In neutral-free-radical implantation experiments where $4 \%$ of the ${ }^{63} \mathrm{Cu}^{-}$ negative ions in the $15 \mathrm{keV}$ beam are photo-deionized using the MPDI, for example, the $\mathrm{S} / \mathrm{N}$ ratio in the measurement of the RBNR flux is as high as 500. Also the high sensitivity of the ICD method was demonstrated by other observations, where the detection limit of the photo-deionized-particle flux was $2 \times 10^{3} \mathrm{~m}^{-2} \mathrm{~s}^{-1}$

As an application of the ICD setup, we attempted to measure the spatial distribution of the number flux of a neutral free-radical beam ( a $15 \mathrm{keV}$ beam of atomic ${ }^{63} \mathrm{Cu}$ in the present case ) by scanning the Faraday cup in the $x y$ plane perpendicular to the ion beam line of the PDINIB apparatus. Figure 3 shows a result of the horizontal scan, obtained under the condition that the light modulation frequency was $1.0 \mathrm{kHz}$ and the time constant of the lock-in amplifier was $10 \mathrm{~s}$. The abscissa $\boldsymbol{x}$ is the displacement of the Faraday-cup aperture from its original position on the NIB axis ( Fig. 1 ). The distorted asymmetric shape of the observed profile was caused by temporal change in conditions of the ion-source plasma. Thus, we can only roughly estimate the width of the neutral free-radical beam from Fig. 3 . After correction of the apparent broadening due to the finite size of the Faraday-cup aperture, the net full-width half-maximum ( FWHM ) at the sample surface is about $2.8 \mathrm{~mm}$, which is slightly greater than the laser beam diameter. This consideration suggests that the neutral free-radical beam obtained under the particular NIB-optics conditions was rather divergent.

The applicability of the ICD method, however, has an intrinsic limitation that the beam kinetic energy of the neutral free radicals has to be at least $100 \mathrm{eV}$. Since the low-energy negative ion beam diverges immediately after passing through its beam waist, it is difficult to accurately monitor the negative ions by a Faraday cup without obstruction of the light path in the MPDI. The PMMP method is more versatile approach, in that it is useful also for the low-energy case, to estimate the total number $G_{r}$ of the neutral free radicals produced per unit time by the PDINIB procedure and to evaluate the photo-deionization efficiency defined as the ratio of $G_{r}$ to $\left|I_{\text {total }} / q\right|$.

\section{CONCLUSIONS}

This article examined performance and an applicability of the ICD method. Judging from the spatial resolution and the convenience, the ICD method seems to be a better choice to evaluate ${ }_{n}$ and than the PMMP method, as long as the beam kinetic energy of the neutral free radicals is higher than $10 \mathrm{keV}$. Although not described here, the high spatial resolution of the ICD setup was confirmed through further experiments, where we succeeded in production of collimated neutral free-radical beams by utilizing the ICD setup as a feedback tool to adjust the NIB optics. These details will be reported in following letters.

\section{ACKNOWLEDGEMENT}

This work is supported by the Ministry of Education, Culture, Sports, Science and Technology, Japan.

\section{REFERENCES}

[1] J. C. Whitehead, Rep. Prog. Phys., 59, 993-1040 (1996).

[2] A. Chutjian and O. J. Orient, Exp. Methods Phys. Sci., 29B, 49-66 (1996).

[3] K. Hayashi, H. Konno, T. Oseki, H. Kojima, and T. Kanayama, Nucl. Instrum. Methods Phys. Res. B, 206C, 403-408 (2003).

[4] K. Hayashi, N. Sakudo, T. Noda, A. Takeda, K. Fujimura, and N. Shimizu, Nucl. Instrum. Methods Phys. Res. B, 127-128, 918-921 (1997).

[5] K. Hayashi, T. Kanayama, T. Shimizu, Y. Kawamura, K. Kameko, and S. Kawakita, J. Vac. Sci. Technol. A, 20, 995-998 (2002).

[6] K. Hayashi, Appl. Phys. Lett., 65, 2084-2086 (1994).

(Received December 9， 2008; Accepted January 5, 2009) 